Wien klin Mag 2019 · 22:204-209 https://doi.org/10.1007/s00740-019-0295-9

Online publiziert: 23. August 2019

(c) Der/die Autor(en) 2019

\section{Birgit Willinger}

Abteilung für Klinische Mikrobiologie, Klinisches Institut für Labormedizin, Medizinische Universität Wien, Wien, Österreich

\section{Moderne Pilzdiagnostik} Fluch oder Segen?
Die Diagnose von invasiven Pilzinfektionen ist nach wie vor schwierig. Sie setzt sich aus mehreren Schritten zusammen und basiert auf der gemeinsamen Bewertung von klinischen Symptomen, radiologischen Befunden, Ergebnissen der histologischen Untersuchung sowie klinisch-chemischen und mikrobiologischen Parametern (• Tab. 1). Erst in der Zusammenschau und gemeinsamen Beurteilung all dieser Befunde ist es möglich, die Diagnose zu stellen. Das klinische Bild ist der erste Hinweis auf das Vorliegen einer Mykose. Während bei oberflächlichen Mykosen die Klinik meist eindeutig ist und der Laborbefund den Verdacht bestätigt, ist bei invasiven Infektionen das klinische Bild vielfach uncharakteristisch. In diesen Fällen sind diagnostische Methoden gefragt, die eine eindeutige Diagnose im Frühstadium der Erkrankung ermöglichen.

\section{Einleitung}

In den vergangenen Jahren wurden viele neue Methoden vorgestellt, von denen auch etliche Einzug ins mikrobiologische Laboratorium gefunden haben. Allerdings stellt sich die Frage, ob bzw. welche von diesen Methoden uns eine rasche, verlässliche und frühzeitige Diagnose ermöglichen. Besonders wertvoll wäre es, wenn es möglich wäre, eine frühzeitige Diagnose mit einer einzigen $\mathrm{Me}$ thode zu stellen.

Goldstandard und daher nach wie vor unerlässlich sind histologische Methoden, die den Beweis einer Infektion liefern. Werden Pilzstrukturen im Gewebe nachgewiesen, gilt das als Beweis für die Infektion. Allerdings ist es vielfach schwierig festzustellen, um welchen Pilz es sich handelt. Zwar ist es noch relativ einfach, zwischen Spross- und Fadenpilzen zu unterscheiden, zwischen den einzelnen Genera finden sich oft nur geringfügige morphologische Unterschiede, sodass eine nähere Bestimmung unmöglich ist. So können beispielsweise Fusarium spp. oder andere Fadenpilze von Aspergillus, der typischerweise dichotome Verzweigungen der Hyphen zeigt, nicht zu unterscheiden sein. Für die Therapie kann es aber entscheidend sein, Genus und sogar Spezies des Pilzes zu kennen. Um zu einer exakten Identifizierung aus Paraffinschnitten zu kommen, können nachfolgend molekularbiologische Verfahren eingesetzt werden.

Das zweite Verfahren, das nach wie vor zum Goldstandard gezählt wird, ist die Kultur. Diese erlaubt die Identifizierung des Erregers bis auf Speziesebene sowie eine Empfindlichkeitstestung. Ungünstig ist allerdings, dass sie vielfach erst nach einigen Tagen positiv wird, die Sensitivität teilweise gering ist, und es nicht immer möglich ist, zwischen Kontami- nation, Besiedelung und Infektion zu unterscheiden. Aus diesem Grund sollten bevorzugt Materialien aus primär sterilen Körperhöhlen zur Untersuchung eingeschickt werden.

\section{Kultur}

Zum Nachweis einer systemischen Infektion werden Blutkulturen eingesetzt. Am häufigsten werden verschiedene Candida-Species, am häufigsten C. albicans, gefolgt von C. glabrata, C. parapsilosis, C. tropicalis, als Erreger von Fungämien in Blutkulturen nachgewiesen. Auch andere Sprosspilze, wie TrichosporonSpecies oder Saccharomyces spp. können bei Immunsupprimierten Ursache einer Fungämie sein. Wesentlich seltener findet man hingegen Fadenpilze in der Blutkultur. Gelingt der Nachweis bei Infektionen mit Fusarium spp. relativ leicht, ist dies bei Aspergillosen wesentlich schwieriger und seltener; bisher ist es in erster Linie A. terreus gewesen, der

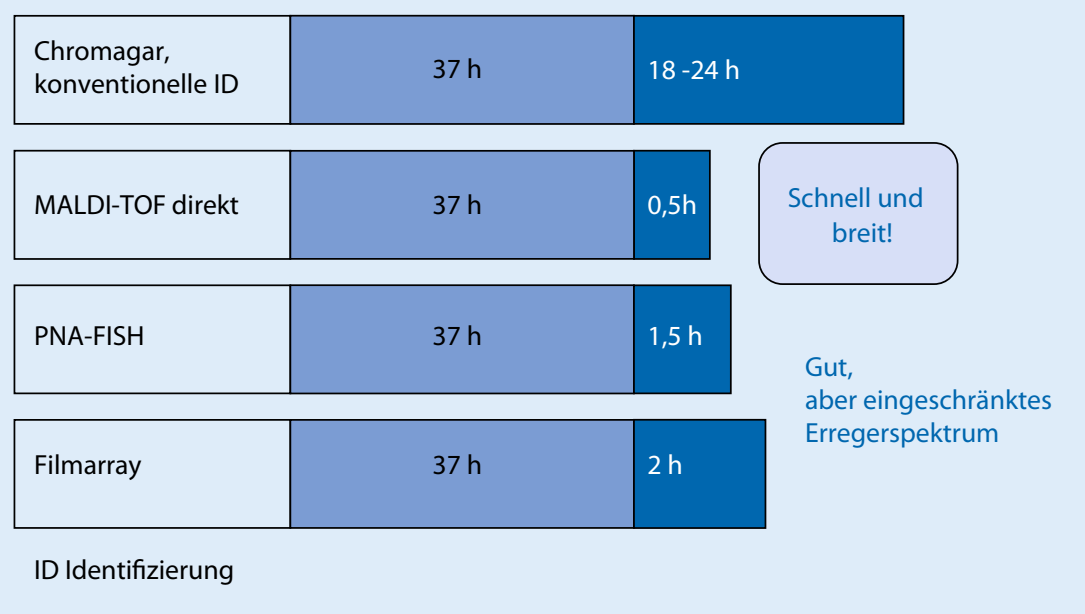

Abb. 1 ॥ Dauer der Identifikation aus der positiven Blutkultur 


\begin{tabular}{|c|c|}
\hline Untersuchungstechnik & Grad der Erregeridentifikation \\
\hline Histologie & Zuordnung in Gruppen \\
\hline Mikroskopie & Zuordnung in Gruppen \\
\hline Kultur & Identifikation bis Speziesebene \\
\hline \multicolumn{2}{|l|}{ Antigennachweis: } \\
\hline Galactomannan & Aspergillus \\
\hline JF5 (Lateral-Flow-Test) & Aspergillus \\
\hline Mannan & Candida \\
\hline 1-3- $\beta$-D-Glucan & $\begin{array}{l}\text { Pilze (Ausnahme: Cryptococcus, Mucormyzeten); keine Differenzie- } \\
\text { rung möglich }\end{array}$ \\
\hline$P C R$ & $\begin{array}{l}\text { Genus- oder speziesspezifisch (abhängig vom Protokoll) } \\
\text { Syndrombasierte Panels mit den häufigsten Erregern }\end{array}$ \\
\hline \multicolumn{2}{|c|}{ JF5 spezieller Aspergillus-Antikörper } \\
\hline
\end{tabular}

\begin{tabular}{|c|c|}
\hline \multirow[t]{5}{*}{ Candida } & Zwei konsekutive Proben zur Beurteilung heranziehen \\
\hline & Bessere Sensitivität als Blutkultur bei tiefer Candidose ( $62 \%$ vs. $17 \%$ ) \\
\hline & $\begin{array}{l}\text { Guter Vorhersagewert bei Blutkultur-negativer intraabdomineller } \\
\text { Candidose }\end{array}$ \\
\hline & $\begin{array}{l}\text { Empfehlung zum Einsatz zur Ausschlussdiagnostik bei systemischen } \\
\text { Candidosen }\end{array}$ \\
\hline & Wertvolle Ergänzung zur konventionellen Diagnostik \\
\hline Pneumocystis-Pneumonie & Auffallend hohe BDG-Titer (>500 pg/ml) \\
\hline
\end{tabular}

in der Blutkultur angezüchtet werden konnte.

Da der Nachweis von Pilzen in der Regel zwischen 20 und $40 \mathrm{~h}$ dauert, ist eine rasche Identifikation des Erregers von großer Bedeutung. Mit konventionellen Methoden zur Identifizierung kann dies bis zu $48 \mathrm{~h}$ (Sprosspilze) oder sogar mehrere Tage (Fadenpilze) dauern. Bis das Ergebnis der Blutkultur vorliegt, können so einige Tage vergehen. Neue Methoden haben hier eine wesentliche Verbesserung gebracht (• Abb. 1). Der Einsatz der Massenspektrometrie (MALDI-TOF MS: „matrix-assisted laser desorption/ ionisation time of flight mass spectrometry") erlaubt eine verlässliche Identifizierung innerhalb von 30 min direkt aus der Blutkultur. Wird eine Blutkultur positiv, dann kann der Erreger innerhalb kurzer Zeit bis auf Speziesebene identifiziert werden. Das Ergebnis steht dem behandelnden Arzt damit bereits am Tag des Positivwerdens zur Verfügung. Das bedeutet einen Zeitgewinn von mindestens $24 \mathrm{~h}$ bis zu mehreren Tagen.

Ähnlich wie MALDI-TOF erlauben auch molekularbiologische Methoden heutzutage eine sehr rasche Identifi- zierung aus der positiven Blutkultur. Bei Nachweis von Sprosspilzen in der Blutkultur bringt der Einsatz der Invitro-Hybridisierung mit fluoreszenzmarkierter peptidischer Nukleinsäure (PNA-Sonden) einen Zeitgewinn um einen Tag. Die Durchführung ist relativ einfach, das Ergebnis liegt nach ca. 1,5h vor. Werden z. B. in der Gram-Färbung einer positiven Blutkultur Sprosspilze gesehen, können nach Anwendung bestimmter Sonden verschiedene CandidaSpecies identifiziert werden. So können beispielsweise C. albicans und C. glabrata von anderen Candida spp. unterschieden werden, was unmittelbare therapeutische Konsequenz hat. Es handelt sich damit um eine hochsensitive und spezifische Methode, deren Einsatz den frühzeitigen Beginn einer gezielten antimykotischen Therapie ermöglicht und damit Therapiekosten drastisch reduzieren könnte.

Filmarrays ermöglichen den gleichzeitigen Nachweis von Bakterien, Viren, Hefen, Parasiten und Resistenzgenen. Es stehen auch Panels für die Identifizierung von Erregern aus positiven Blutkulturen zur Verfügung, die eine hohe Sensitivität und Spezifität aufweisen. Bei minima-
Tab. 2 Eigenschaften BDG-Nachweis

Panfungaler Marker ohne Differenzierung

Kein Nachweis von Mucormyzeten und Cryptococcus

Häufig falsch-positive Ergebnisse durch:

Menschliche Blutprodukte

Hämodialyse

Hohe Triglyceride

Gaze

Glucan-haltige Materialien

ler Bearbeitungszeit (ca. 2 min) liegt das Ergebnis bereits nach einer Stunde vor.

$\mathrm{Zu}$ beachten ist jedoch, dass ausschließlich im Panel enthaltene Erreger nachgewiesen werden können. Bei einem negativen Ergebnis müssen daher andere Methoden eingesetzt werden.

Alle genannten Verfahren bringen zwar eine Verbesserung in der Identifizierung des Erregers, trotzdem bleibt der Umstand, dass diese nur bei einer positiven Blutkultur eingesetzt werden können, der größte Nachteil, da dies mit einem Zeitverlust von etlichen Stunden bis zu mehreren Tagen einhergeht.

\section{Einsatz von Biomarkern}

In den vergangenen Jahren wurde dem Antigennachweis mittels ELISA mehr Beachtung eingeräumt. So gibt es unterschiedliche Testkits, die den Nachweis von Candida-Antigen erlauben. Vor allem die Kombination von Antikörper- und Antigennachweis konsekutiv abgenommener Seren bei Hochrisikopatienten ermöglicht es, die Diagnose invasiver Candidosen rascher zu stellen und kann als wertvolle Zusatzuntersuchung angesehen werden.

Zum Nachweis der invasiven Aspergillose steht seit Längerem ein kommerziell erhältlicher Test (Platelia Aspergillus, Bio-Rad, USA) zur Verfügung, der zirkulierendes Galactomannan, ein Bestandteil der Zellwand von Aspergillus spp., erfasst. Dieser Assay hat besonders als Screeningtest bei hämatologischen Patienten einen hohen Stellenwert. Er kann sowohl zur Frühdiagnose als auch zum 
Therapiemonitoring eingesetzt werden. Wird der Test zum Screening eingesetzt, wird empfohlen, ihn mindestens zweimal pro Woche durchzuführen, damit eine ausreichende Sensitivität gewährleistet ist. Damit soll auch eine möglichst frühzeitige und effiziente Therapie ermöglicht werden. In einer Metaanalyse wurde gezeigt, dass der Test einen ausgezeichneten negativen Vorhersagewert und eine exzellente Spezifität aufweist, d.h. bei Vorliegen eines negativen Ergebnisses eine invasive Aspergillose ausgeschlossen werden kann.

$\mathrm{Zu}$ beachten ist, dass falsch-positive Ergebnisse, wie z.B. durch Antibiotika wie Piperacillin/Tazobactam oder Amoxicillin mit und ohne Clavulansäure oder durch Kreuzreaktionen mit anderen Schimmelpilzen, auftreten können. Wiederholte Testungen sind daher umso wichtiger, um falsch-positive Ergebnisse $\mathrm{zu}$ minimieren. Mittlerweile ist der Nachweis auch aus bronchoalveolärer Lavage (BAL) zugelassen. In mehreren Studien wurde gezeigt, dass sowohl Sensitivität als auch Spezifität höher sind als im Serum. Das wurde sowohl bei hämatologischen als auch bei organtransplantierten Patienten beobachtet. Auch bei Intensivpatienten und Organtransplantierten war die Spezifität des Galactomannan-Nachweis in der BAL höher als im Serum oder die Kultur. Zusätzlich ist $\mathrm{zu}$ beachten, dass auch eine Besiedelung des Respirationstrakts mit Aspergillus spp. zu positiven Testergebnissen führen kann. Da eine solche Besiedelung jedoch bei hämatologischen und organtransplantierten Patienten unerwünscht ist, sollten diese Ergebnisse dazu verwendet werden, mit weiteren Untersuchungen entweder eine invasive Aspergillose auszuschließen oder mit einer antifungalen Prophylaxe/Therapie zu beginnen.

Beim Einsatz dieses Tests ist zu beachten, dass der alleinige Antigennachweis für eine exakte Diagnose nicht ausreichend ist. Die Ergebnisse müssen immer in Zusammenschau mit Klinik und anderen erhobenen Befunden beurteilt werden. Um eine maximale Sensitivität des Tests zu erzielen, sollte die Abnahme des Untersuchungsmaterials immer vor Beginn einer antifungalen Therapie erfol-

Wien klin Mag 2019·22:204-209 https://doi.org/10.1007/s00740-019-0295-9

(c) Der/die Autor(en) 2019

\section{B. Willinger}

\section{Moderne Pilzdiagnostik. Fluch oder Segen?}

\section{Zusammenfassung}

Die Diagnose von invasiven Pilzinfektionen ist nach wie vor schwierig. Sie setzt sich aus mehreren Schritten zusammen und basiert auf der gemeinsamen Bewertung von klinischen Symptomen, radiologischen Befunden, Ergebnissen der histologischen Untersuchung sowie klinisch-chemischen und mikrobiologischen Parametern. Erst in der Zusammenschau und gemeinsamen Beurteilung all dieser Befunde ist es möglich, die Diagnose zu stellen. Neue Entwicklungen vor allem auf dem Gebiet der molekularbiologischen Techniken wie auch der
Einsatz von Biomarkern bei immunologischserologischen Untersuchungen haben wesentliche Verbesserungen im Sinne einer frühzeitigen und raschen Diagnostik gebracht. Trotzdem ist es nach wie vor notwendig, unterschiedliche Testverfahren zu kombinieren, um zu einer verlässlichen Diagnose zu kommen.

Schlüsselwörter

Biomarker - Galactomannan · Beta-D-Glucan . Molekulare Assays · T2 Candida · Filmarray

\section{Modern Fungal Diagnostics. Curse or Blessing?}

\section{Abstract}

The diagnosis of invasive fungal infections remains difficult. It consists of several steps and is based on the joint evaluation of clinical symptoms, radiological findings, histological examination results, clinicochemical and microbiological parameters. A diagnosis can first be made by a synopsis and total assessment of all these findings. New developments, especially in the field of molecular biological techniques and the use of biomarkers in immunological and serological investigations have resulted in significant improvements in terms of early and rapid diagnosis. Nevertheless, the combination of various test procedures is mandatory to enable earlier and reliable diagnosis of systemic fungal infections.

\section{Keywords}

Biomarker - Galactomannan - Beta-D-glucan Molecular assays · T2Candida · Filmarray gen. Unter der Therapie kann die Sensitivität drastisch sinken und somit die Aussagekraft des Tests stark einschränken. Positive Ergebnisse müssen stets wiederholt werden. Erst bei Vorliegen zweier positiver Ergebnisse kann dies als Hinweis auf das Vorliegen einer invasiven Aspergillose angesehen werden. Dieser Test ist auch für ein Therapiemonitoring geeignet, da beim Ansprechen auf die antifungale Therapie eine Abnahme des Antigentiters zu beobachten ist.

Seit Kurzem stehen zwei immunologische Tests (Lateral-Flow-Tests) zum Nachweis von unterschiedlichen Aspergillus-Antigenen zur Verfügung. Ein Test weist Galactomannan nach (Immy, OK, USA), ein weiterer erfasst JF5 (OLM Diagnostics, Newcastle, UK), ein extrazelluläres Glykoprotein, das ausschließlich während des aktiven Wachstums von $A s$ pergillus spp. sezerniert wird. Beide Tests sind einfach durchzuführen und liefern innerhalb kurzer Zeit Ergebnisse. Durch das ausschließliche Erfassen auskeimender Konidien von Aspergillus mittels JF5Test werden Besiedelung und Kontamination das Testergebnis nicht verfälscht. Da Kreuzreaktionen keinen großen Einfluss zu haben scheinen, könnte dieser Test eine wertvolle Ergänzung in der Diagnostik von invasiven Aspergillosen darstellen. Klinische Studien sollen nun den Einsatz dieser Tests evaluieren.

1-3- $\beta$-D-Glucan (BDG) ist ein Zellwandbestandteil vieler Spross- und Fadenpilze und kann während invasiver Mykosen im Blut detektiert werden. Die Angaben zu Sensitivität und Spezifität schwanken abhängig von den verschiedenen Studien und liegen zwischen 50 und $100 \%$. Neben der invasiven Candidose und Aspergillose werden auch Infektionen durch Fusarium, Trichosporon, 


\begin{tabular}{ll}
\hline T2 Candida & Blutkultur \\
\hline Rasche Ergebnisse & Langsam (mind. 18h bis mehrere Tage) \\
\hline $\begin{array}{l}\text { Hohe Sensitivität und Spezifität } \\
\text { Keine Beeinflussung durch antifungale Therapie }\end{array}$ & Sensitivität 50-75\% \\
$\begin{array}{l}\text { Ausschließlicher Nachweis von Spezies, die im Panel enthalten sind } \\
\text { Keine weitere Differenzierung (z. B. C.-parapsilosis-Komplex) }\end{array}$ & Sensitivität wird durch antifungale Therapie reduziert \\
\hline $\begin{array}{l}\text { Keine Empfindlichkeitsprüfung möglich } \\
\text { Hohe Kosten }\end{array}$ & Identifikation aller Isolate \\
\hline Vorteile jeweils kursiv hervorgehoben & Empfindlichkeitsprüfung möglich \\
\hline
\end{tabular}

Tab. 5 Vergleich von nichtkulturbasierten mit konventionellen Methoden

\begin{tabular}{|c|c|c|c|c|c|}
\hline & BDG & T2 Candida & PNA-FISH & Filmarray & MALDI-TOF MS \\
\hline Kultur & $0 \mathrm{~h}$ & $0 \mathrm{~h}$ & $19-75 \mathrm{~h}$ & 19-75h & 19-75h \\
\hline $\begin{array}{l}\text { Dauer bis } \\
\text { Ergebnis }\end{array}$ & $40 \mathrm{~min}$ & $180-240 \mathrm{~min}$ & $90 \mathrm{~min}$ & $60 \mathrm{~min}$ & $30 \mathrm{~min}$ \\
\hline \multirow[t]{4}{*}{ Vorteile } & Rasch & Rasch & Rasch & Rasch & Rasch \\
\hline & \multirow[t]{3}{*}{ Panfungal } & Hohe Treffsicherheit & \multirow[t]{3}{*}{$\begin{array}{l}\text { Hohe } \\
\text { Treffsicherheit }\end{array}$} & $\begin{array}{l}\text { Hohe Treffsicher- } \\
\text { heit }\end{array}$ & Hohe Treffsicherheit \\
\hline & & Sehr niedriges Detektionslimit & & \multirow{2}{*}{$\begin{array}{l}\text { Einfache Durch- } \\
\text { führung ( } 2 \mathrm{~min})\end{array}$} & ID bis auf Speziesebene \\
\hline & & $(1-3 \mathrm{KBE} / \mathrm{ml})$ & & & Geringe Reagenzienkosten \\
\hline \multirow[t]{4}{*}{ Nachteile } & $\begin{array}{l}\text { Keine ID des nachge- } \\
\text { wiesenen Erregers }\end{array}$ & $\begin{array}{l}\text { Nachweis von nur } 5 \text { Candida-Ar- } \\
\text { ten }\end{array}$ & $\begin{array}{l}\text { Eingeschränktes } \\
\text { Erregerspektrum }\end{array}$ & $\begin{array}{l}\text { Eingeschränktes } \\
\text { Erregerspektrum }\end{array}$ & $\begin{array}{l}\text { Ergebnis abhängig von Daten- } \\
\text { bank }\end{array}$ \\
\hline & \multirow{3}{*}{$\begin{array}{l}\text { Kontaminationsgefahr } \\
\text { mit hoher Rate von } \\
\text { falsch positiven } \\
\text { Ergebnissen }\end{array}$} & $\begin{array}{l}\text { Aussagekraft abhängig von der } \\
\text { lokalen Prävalenz }\end{array}$ & \multirow{3}{*}{$\begin{array}{l}\text { Kann nur aus } \\
\text { positiver Blutkultur } \\
\text { durchgeführt } \\
\text { werden }\end{array}$} & \multirow{3}{*}{$\begin{array}{l}\text { Kann nur aus } \\
\text { positiver Blutkultur } \\
\text { durchgeführt } \\
\text { werden }\end{array}$} & $\begin{array}{l}\text { Kann nur aus positiver Blutkul- } \\
\text { tur durchgeführt werden }\end{array}$ \\
\hline & & Hohe Kosten & & & Hohe Instrumentenkosten \\
\hline & & $\begin{array}{l}\text { Evaluierung nur bei Candidämie } \\
\text { und systemischer Candidose }\end{array}$ & & & \\
\hline
\end{tabular}

Saccharomyces und Acremonium erfasst. Der Nachweis von Pneumocystis jirovecii mittels BDG ist besonders gut evaluiert und stellt eine wertvolle Unterstützung bei der Diagnose einer PneumocystisPneumonie dar. $\mathrm{Zu}$ beachten ist allerdings, dass es bei positivem Testausfall nicht möglich ist, die infektionsauslösende Pilzgattung zu erkennen. Im Gegensatz zum Candida-Antigennachweis fällt der Nachweis von BDG bei oraler Candidose oder Kolonisation negativ aus. Infektionen durch C. neoformans und $\mathrm{Mu}$ cormyzeten werden durch diesen Test nicht erfasst. Falsch-positive Reaktionen wurden bei Patienten mit Hämodialyse, Zirrhose sowie nach bauchchirurgischen Eingriffen beobachtet ( $\bullet$ Tab. 2 und 3).

Mittlerweile hat sich gezeigt, dass dieser Test besonders gut zum Ausschluss einer invasiven Pilzinfektion geeignet ist und damit auch im Sinne einer zielgerichteten Therapie sowie des antimikrobiellen Stewardships einge- setzt werden kann. So konnte in einigen Studien gezeigt werden, dass der Einsatz dieses Tests zur Früherkennung möglicher Candida-Infektionen besonders erfolgreich sein könnte. Somit wäre es z.B. möglich, Echinocandine gezielter einzusetzen, ohne dass die Gesamtletalität gesteigert würde. Gleichzeitig würde das auch eine Reduktion der Behandlungskosten bedeuten. Mittlerweile gibt es mehrere Anbieter von Testkits, die auf dem Nachweis von BDG basieren. Eine endgültige Evaluation dieser Kits liegt noch nicht vor.

\section{Molekularbiologische Assays}

Der Einsatz molekularer Techniken soll einen möglichst raschen und sensitiven Erregernachweis erlauben. Die meisten Techniken basieren auf dem Prinzip der Polymerasekettenreaktion (PCR) und beruhen damit auf einer gezielten Vermehrung von für bestimmte
Krankheitserreger spezifischen DNAoder RNA-Abschnitten. Neue Methoden geben zusätzlich Auskunft über die Menge des nachgewiesenen Erregers in der Patientenprobe (quantitative Realtime-PCR). Diese Methoden können sowohl zum Erregernachweis als auch zur Identifikation des Erregers verwendet werden, das Ergebnis liegt meist innerhalb weniger Stunden vor.

Diese Formen der PCR haben sich als sehr sensitiv herausgestellt und könnten je nach Auswahl der Gensequenz für die Diagnose von Aspergillosen, Candidosen oder anderen Pilzinfektionen eingesetzt werden. Dabei werden entweder speziesspezifische Primer verwendet, mit denen der Nachweis nur eines bestimmten Pilzes möglich ist, oder sog. panfungale Primer, die eine größere Breite von Pilzen nachweisen. Immer häufiger werden auch syndromorientierte Panels angeboten, die z. B. bei Sepsis, Infektionen des Respirationstrakts oder Infektionen 
des Urogenitaltrakts die häufigsten Erreger nachweisen. Diese Panels werden zwar in gewissen Bereichen bereits eingesetzt, befinden sich aber vielfach noch in Entwicklung oder klinischer Evaluierung. Es ist $\mathrm{zu}$ erwarten, dass sie sich hilfreich in das diagnostische Spektrum einbringen werden. $\mathrm{Zu}$ beachten ist, dass negative Ergebnisse nicht eine Pilzinfektion ausschließen, sondern nur bedeuten, dass kein im Panel enthaltener Erreger nachgewiesen werden konnte.

Generell gilt, dass alle molekularbiologischen Assays noch besser klinisch evaluiert werden müssen und die Interpretation der Ergebnisse zurzeit nicht immer eindeutig möglich ist. Vielfach wird für Tests, die zielgerichtet auf eine kleine Erregergruppe abgestimmt sind, eine hohe Sensitivität mit hoher negativer Aussagekraft beschrieben. Das bedeutet, dass negative Ergebnisse in hohem Maße verlässlich sind. Positive Ergebnisse sind hingegen wesentlich schwieriger $\mathrm{zu}$ beurteilen, da in allen Studien nur eine geringe Anzahl von bewiesenen und wahrscheinlichen $\mathrm{My}$ kosen eingeschlossen werden konnte. Daher ist es besonders wichtig, alle verfügbaren klinischen Informationen für die endgültige Interpretation des Testergebnisses heranzuziehen. Bereits die Auswahl des Untersuchungsmaterials kann gravierende Auswirkungen haben. Wird das Material vom Infektionsort direkt entnommen, ist mit einer höheren Pilzlast als im Blut zu rechnen, weswegen der Nachweis im Blut wahrscheinlich seltener als im Gewebe gelingt. Eine bereits laufende antimykotische Therapie kann ebenfalls die Pilzlast reduzieren, wodurch bei manchen Assays der DNANachweis erschwert werden kann. Vollkommen unklar ist die Interpretation von singulären und intermittierend positiven PCR-Ergebnissen bei konsekutiv abgenommenen Proben. Niemand weiß, was es bedeutet, wenn von mehreren Proben einige positive Ergebnisse liefern, die anderen jedoch negativ ausfallen. Möglicherweise sind diese positiven Ergebnisse durch eine Kontamination oder durch eine transiente Besiedelung des Patienten verursacht und klinisch nicht relevant. Es wäre jedoch auch möglich, dass bereits frühe Krankheitsstadien mit unterschiedlich hoher Pilzlast unterschiedlich gut erfasst werden. Auch eine antimykotische Therapie könnte zu intermittierend positiven Ergebnissen führen.

Eine neu eingeführte Methode ist die T2-Magnetresonanz (T2MR, T2 BioSystems Inc., MA, USA). T2MR ist ein vollautomatisiertes Gerät, das die Identifizierung von fünf Candida spp. aus Vollblut ermöglicht. Dabei werden C. albicans, C. tropicalis, C. glabrata, C. krusei und C. parapsilosis-Komplex erfasst. Die Durchführung des Tests sowie die Bedienung des Geräts sind einfach und wenig zeitaufwändig. Bereits nach drei bis fünf Stunden liegt das Ergebnis vor. Die Aussagekraft des Tests hängt von der Prävalenz der Candidosen im jeweiligen Bereich ab. Je höher die Prävalenz, desto besser ist vor allem der positive Vorhersagewert. Die ersten Studien zeigten eine sehr hohe Sensitivität, verbunden mit hoher Spezifität, sodass der Einsatz dieser Technik sowohl eine bessere Nachweisrate als auch raschere Ergebnisse erlaubt. Dies wiederum ermöglicht eine raschere zielgerichtete Therapie oder eine individuell gesteuerte Therapiedauer und könnte so zum besseren Heilungserfolg beitragen. Vor- und Nachteile im Vergleich zur Kultur sind in $\bullet$ Tab. 4 dargestellt.

\section{Fazit}

All diese Neuerungen bieten einen großen Fortschritt in der Diagnose von invasiven Pilzinfektionen und stellen damit eine segensreiche Entwicklung dar. Auf der anderen Seite bietet keiner dieser Tests die Möglichkeit, Pilzinfektionen ohne Bestimmung weiterer Parameter mit $100 \%$ Sicherheit zu diagnostizieren (•Tab. 5). Falsch-positive sowie falsch-negative Ergebnisse können die richtige Interpretation der Ergebnisse erschweren. Damit die segensreichen Entwicklungen nicht zum Fluch werden, ist genaues Wissen über die Aussagekraft der einzelnen Testverfahren Voraussetzung. Nur so gelingt es den richtigen Einsatz festzulegen und die Ergebnisse richtig zu interpretieren. Trotz dieser breiten $\mathrm{Pa}$ lette an diagnostischen Tests muss nach wie vor eine Kombination verschiedener Techniken und Untersuchungsme- thoden eingesetzt werden, um zu einer verlässlichen Diagnose zu kommen.

\section{Korrespondenzadresse}

a.o. Univ.-Prof. Dr. Birgit Willinger

Abteilung für Klinische Mikrobiologie,

Klinisches Institut für Labormedizin,

Medizinische Universität Wien

Währinger Gürtel 18-20/5P, 1090 Wien

Österreich

birgit.willinger@meduniwien.ac.at

Funding. Open access funding provided by Medica University of Vienna.

\section{Einhaltung ethischer Richtlinien}

Interessenkonflikt. B. Willinger gibt an, dass kein Interessenkonflikt besteht.

Für diesen Beitrag wurden von den Autoren keine Studien an Menschen oder Tieren durchgeführt. Für die aufgeführten Studien gelten die jeweils dort angegebenen ethischen Richtlinien.

Open Access. Dieser Artikel wird unter der Creative Commons Namensnennung 4.0 International Lizenz (http://creativecommons.org/licenses/by/4.0/deed. de) veröffentlicht, welche die Nutzung, Vervielfältigung, Bearbeitung, Verbreitung und Wiedergabe in jeglichem Medium und Format erlaubt, sofern Sie den/die ursprünglichen Autor(en) und die Quelle ordnungsgemäßnennen, einen Linkzur Creative Commons Lizenz beifügen und angeben, ob Änderungen vorgenommen wurden.

Hinweis des Verlags. Der Verlag bleibt in Hinblick auf geografische Zuordnungen und Gebietsbezeichnungen in veröffentlichten Karten und Institutsadressen neutral. 
Hier steht eine Anzeige.

\section{曾 Springer}

